\title{
Desenvolvimento e avaliação de usabilidade do aplicativo móvel pediálise para ensino e avaliação de pacientes pediátricos em diálise
}

\author{
Development and usability evaluation of the pedialysis mobile application for teaching and \\ evaluation of pediatric patients on dialysis \\ Evaluación del desarrollo y usabilidad de la aplicación móvil de pediálisis para la enseñanza y \\ evaluación de pacientes pediátricos en diálisis
}

Lia Cordeiro Bastos Aguiar ORCID: https://orcid.org/0000-0002-4229-7311 Centro Universitário Christus, Brasil

E-mail: liacbastos@yahoo.com.br

Camila Ximenes Pinto

ORCID: https://orcid.org/0000-0001-8854-8625 Centro Universitário Christus, Brasil E-mail: camilaximenesp@gmail.com Maria Améllya Nunes Diniz ORCID: https://orcid.org/0000-0002-1883-202X Centro Universitário Christus, Brasil E-mail: mariaamellyand@gmail.com

Ana Adélia Sá Costa ORCID: https://orcid.org/0000-0001-9242-6612 Centro Universitário Christus, Brasil

E-mail: sc.adelia@gmail.com

Edgar Marçal de Barros Filho ORCID: https://orcid.org/0000-0001-5037-2724 Universidade Federal do Ceará, Brasil E-mail: edgar@virtual.ufc.br

Melissa Soares Medeiros ORCID: https://orcid.org/0000-0002-5881-1485 Hospital São José de Doenças Infecciosas, Brasil E-mail: melmedeiros@hotmail.com

Jayanne Antonia Ferreira Rabelo ORCID: https://orcid.org/0000-0003-4419-2166 Centro Universitário Christus, Brasil E-mail: jayannerabelo@hotmail.com

\begin{abstract}
Resumo
A prevalência de doença renal crônica (DRC) aumentou mundialmente, assim como os custos para o tratamento adequado. A escolha do método dialítico entre hemodiálise e diálise peritoneal depende de variáveis como idade e peso, condições hemodinâmicas, presença de cirurgias abdominais prévias ou doenças graves abdominais atuais, além da disponibilidade da terapia no hospital e capacitação dos profissionais. A utilização de aplicativos surge como auxílio, minimizando falhas no diagnóstico, bem como ferramenta importante na conduta adequada de seleção do cateter e vaso apropriado, estudo das causas de insuficiência renal, seguimento de exames importantes e terapia antimicrobiana racional. Desenvolvemos um aplicativo móvel nacional, para sistema Android, que funciona sem conexão à internet e custos ao usuário, para acompanhamento de DRC e diálise em pediatria, direcionado para ensino de estudantes de medicina. Para avaliação da usabilidade, utilizamos a escala SUS (System Usability Scale) aplicada a 9 alunos e 12 professores, além de 16 alunos que responderam ao caso sem uso do aplicativo (controles). O projeto foi submetido via plataforma Brasil e aprovado pelo Comitê de Ética e Pesquisa da Instituição. Um Termo de Consentimento Livre e Esclarecido (TCLE) foi aplicado em todos os participantes. A análise dos dados demonstrou uma escala SUS média de 74,5 pontos, considerada excelente em termos de interface. O aplicativo foi também avaliado de forma qualitativa, sendo elogiado pelos usuários. Conclui-se que o aplicativo apresentou bons resultados de usabilidade, podendo auxiliar alunos e profissionais no ensino de nefropediatria.
\end{abstract}

Palavras-chave: Hemodiálise; Aplicativo móvel; Diálise peritoneal; Infecção relacionada a diálise.

\footnotetext{
Abstract

The prevalence of chronic kidney disease (CKD) has increased worldwide, as well as the costs of adequate treatment. The choice of dialysis method between hemodialysis and peritoneal dialysis depends on variables such as age and
} 
weight, hemodynamic conditions, presence of previous abdominal surgery or current severe abdominal diseases, in addition to the availability of therapy in the hospital and training of professionals. The use of apps appears as an aid, minimizing misdiagnosis, as well as an important tool to the proper conduct of selection of the appropriate catheter and vessel, study of the causes of renal failure, follow-up of important exams and rational antimicrobial therapy. We developed a national mobile application, for Android system, which works without internet connection and has no cost to the user, for monitoring CKD and dialysis in pediatrics, aimed at teaching medical students. To assess usability, we used the SUS scale (System Usability Scale) applied to 9 students and 12 teachers, in addition to 16 students who responded to the case without using the application (controls). The project was submitted via the Brazil platform and approved by the Institution's Ethics and Research Committee. An Informed Consent Form (ICF) was applied to all participants. Data analysis showed an average SUS scale of 74,5 points, considered good in terms of interface. The application was also well evaluated, being praised by users. It is concluded that the application presented excellent usability results, being able to help students and professionals in the teaching of nephropediatrics.

Keywords: Hemodialysis; Mobile App; Peritoneal Dialysis; Dialysis-related infection.

\begin{abstract}
Resumen
La prevalencia de la enfermedad renal crónica (ERC) ha aumentado en todo el mundo, al igual que los costos de un tratamiento adecuado. La elección del método de diálisis entre hemodiálisis y diálisis peritoneal depende de variables como la edad y el peso, las condiciones hemodinámicas, la presencia de cirugía abdominal previa o enfermedades abdominales graves actuales, además de la disponibilidad de terapia en el hospital y la formación de los profesionales. El uso de aplicaciones aparece como una ayuda, minimizando los diagnósticos erróneos, así como una herramienta importante en la correcta realización de la selección del catéter y vaso adecuados, el estudio de las causas de la insuficiencia renal, el seguimiento de exámenes importantes y la terapia antimicrobiana racional. Desarrollamos una aplicación móvil nacional, para el sistema Android, que funciona sin conexión a internet y sin costos de usuario, para el seguimiento de la ERC y diálisis en pediatría, dirigida a la docencia de estudiantes de medicina. Para evaluar la usabilidad se utilizó la escala SUS (Escala de usabilidad del sistema) aplicada a 9 estudiantes y 12 docentes, además de 16 estudiantes que respondieron al caso sin utilizar la aplicación (controles). El proyecto fue presentado a través de la plataforma Brasil y aprobado por el Comité de Ética e Investigación de la Institución. Se aplicó un formulario de consentimiento informado (FICF) a todos los participantes. El análisis de datos mostró una escala SUS promedio de 75 puntos, considerada excelente en términos de interfaz. La aplicación también fue evaluada cualitativamente, siendo elogiada por los usuarios. Se concluye que la aplicación presentó excelentes resultados de usabilidad, pudiendo ayudar a estudiantes y profesionales en la enseñanza de la nefropediatría.
\end{abstract}

Keywords: Hemodiálisis; Aplicación móvil, Diálisis peritoneal; Infección relacionada con la diálisis.

\title{
1. Introdução
}

A doença renal crônica terminal (DRCT) é um problema de saúde pública de grande importância e há evidências de aumento na incidência e na prevalência dessa condição clínica. Tal doença implica em custos elevados para o sistema de saúde pela complexidade de recursos terapêuticos necessários, além de aumentar a morbidade e mortalidade dos pacientes, notadamente por eventos cardiovasculares. Em crianças e adolescentes, essas dificuldades são ainda mais importantes, dadas as peculiaridades de cada faixa etária, fazendo com que o tratamento desses pacientes seja quase individualizado e, consequentemente, mais complexo e dispendioso (Nogueira, 2011).

A diálise é um termo genérico que se refere a um procedimento artificial cujo objetivo é remover uma série de substâncias tóxicas retidas no organismo de indivíduos que apresentam determinadas patologias que cursam com insuficiência renal, tendo como finalidade a retirada de líquidos em excesso e de produtos metabólicos do organismo, bem como a possibilidade de correção de distúrbios hidroeletrolíticos e do pH. Há dois tipos principais de diálise: a hemodiálise (HD) e a diálise peritoneal (DP). A escolha do método dialítico depende de variáveis como idade e peso do paciente, condições hemodinâmicas, presença de cirurgias abdominais prévias ou doenças graves abdominais atuais, além da disponibilidade da terapia no hospital e capacitação dos médicos responsáveis.

A hemodiálise é um procedimento no qual o sangue é removido do corpo, encaminhado para um dialisador que filtra os resíduos metabólicos e é devolvido para o paciente o sangue purificado, devendo ser realizado, no mínimo, 3 vezes por semana em um período de 4 horas. Esse procedimento depende da presença de um acesso vascular, que pode ser obtido através de uma fístula arteriovenosa ou através de um cateter de curta ou de longa permanência. O objetivo da terapia hemodialítica é a 
eliminação do excesso de substâncias tóxicas e de fluídos em excesso que acabam se acumulando em pacientes portadores de doenças renais.

Apesar de comumente ser uma opção para início da diálise ou para a transição entre diferentes tipos de acessos, é importante ressaltar que o cateter de hemodiálise está relacionado com várias complicações que podem ocorrer durante ou imediatamente após a sua colocação. Entre as complicações de curto prazo estão o pneumotórax, o hemotórax, a punção arterial, o sangramento, as arritmias, a embolia pulmonar e a laceração das veias centrais. Já as complicações de longo prazo estão relacionadas com infecções, mau funcionamento do cateter, bainha de fibrina, trombose, má posição da ponta do cateter e estenose venosa central. Assim, seria interessante que os profissionais que usam esses dispositivos compreendam o método de inserção de forma segura, reconhecendo as complicações e a adesão das diretrizes elaboradas para proteger os pacientes (Bream, 2016).

Outro método de diálise disponível é a diálise peritoneal, na qual o processo ocorre dentro do corpo do paciente, com auxílio de uma membrana natural semipermeável que substitui a função de filtração do rim. O peritônio separa o sangue do paciente da solução de diálise, permitindo a passagem de pequenas partículas e de líquido, tal qual o capilar da hemodiálise. O líquido de diálise é inserido na cavidade peritoneal através de um cateter, permitindo que as trocas aconteçam, e sendo posteriormente drenado através do mesmo cateter.

O sucesso da diálise peritoneal como terapia de substituição renal depende de um bom funcionamento do cateter peritoneal. Assim, o conhecimento das melhores práticas na inserção do cateter pode minimizar o risco de complicações do cateter que causam a falha da diálise peritoneal e otimizar a terapia, (Crabtree, 2017), além de reduzir a morbidade do paciente e aumentar a sobrevivência ao transplante renal (Imani, 2018).

Um estudo retrospectivo executado nos United States Renal Database System (USRDS) abordando pacientes menores de 21 anos que realizaram tratamento com hemodiálise e de diálise peritoneal concluiu que não houve diferença na mortalidade em crianças com menos de treze anos. No entanto, a diálise peritoneal foi associada a maior mortalidade em pacientes com treze anos ou mais. Esse estudo também reconheceu que vários fatores, como idade, qualidade de vida e acesso às instalações de saúde, podem contribuir para a escolha do método de diálise. Assim, a HD continua a ser a opção de diálise mais prevalecente para crianças e adolescentes nos EUA (63\%), em relação à DP (Arhuidese, 2020).

De acordo com o estudo do The North American Pediatric Renal Trials and Collaborative Studies (NAPRTCS) sobre pacientes pediátricos com doença renal, há uma maior probabilidade de o tratamento ser iniciado em diálise peritoneal na faix a etária neonatal (98\% dos neonatos) do que em hemodiálise (Carey, 2015). Assim, a diálise peritoneal é um método comumente usado para suporte renal em pacientes pediátricos, entretanto pode ser associada com o risco de complicações pós-cirúrgicas, como o deslocamento ou a migração do cateter, o vazamento no local do cateter, o desenvolvimento de peritonite e de hérnias abdominais, a erosão do cateter e as aderências relacionadas para o cateter. Tais problemas podem resultar em uma falha no funcionamento adequado do cateter, bem como na necessidade de substituição. (Laplant, 2018).

A inserção do cateter de diálise peritoneal pode ser associada com várias complicações importantes do procedimento, além de complicações relacionadas à anestesia. Tais complicações incluem vazamento, hemorragia, lesão visceral e disfunção do fluxo mecânico. (Crabtree, 2017).

Complicações repetidas relacionadas ao cateter peritoneal, como as infecções, lesam a membrana peritoneal e dificultam o processo de cicatrização, o que resulta em uma diálise menos eficaz, uma disfunção do peritônio, e, potencialmente, uma falha no funcionamento da membrana (Szeto, 2002). A peritonite, principal complicação infecciosa dos pacientes pediátricos que realizam a diálise peritoneal, pode corresponder à principal causa de hospitalização em decorrência desse procedimento, bem como representar uma taxa de mortalidade de até $10 \%$. Esse processo infeccioso pode desencadear repercussões negativas tanto 
a curto prazo quanto a longo prazo, por exemplo, a redução da qualidade de vida, a necessidade de troca da técnica de diálise, a desnutrição e a obstrução intestinal devido à fibrose da membrana peritoneal (Rivacoba, 2018).

A escolha do tamanho do cateter adequado, o procedimento de colocação do mesmo, a identificação do vaso sanguíneo mais adequado para implante nas crianças são pontos fundamentais para que a técnica ocorra de maneira adequada, evitando complicações. Assim, o pré-operatório é uma etapa instrumental fundamental para facilitar a realização do procedimento, evitando eventos adversos e promovendo o resultado desejado. (Crabtree, 2017).

Percebe-se que, muitas vezes, em uma mesma equipe, diferentes profissionais envolvidos no tratamento de um paciente apresentam condutas diversas em relação aos cuidados com a sua saúde. O uso do aplicativo é também, uma oportunidade para uniformizar e oficializar as condutas no cuidado ao paciente (Echer, 2005). Assim, a utilização da tecnologia digital surge como potencial instrumento no auxílio para orientar sobre qual o melhor cateter para a faixa etária, qual a melhor técnica de inserção desse cateter e quais os melhores locais de punção, levando em conta a complicação de cada terapia, o que iria resultar em uma melhora do tratamento desses indivíduos. Além disso, o médico poderia escolher com maior segurança o cateter e o vaso apropriado, bem como antibiótico mais adequado para o tratamento das infecções, etc.

O uso da tecnologia na educação médica chegou de modo disruptivo, auxiliando a aprendizagem de estudantes, internos, residentes e médicos na prática diária. Entretanto seu uso deve ser ferramenta auxiliar, não deve ser substituto para o aprendizado face a face. A utilização por parte do docente deve ocorrer de maneira planejada, estratégica e responsável, com adequação ao currículo, buscando atingir os objetivos educacionais, sendo uma ferramenta educacional e não o centro da ação (Martins, 2017).

\section{Metodologia}

Trata-se de um estudo exploratório, observacional, descritivo, de abordagem quantitativa e de levantamento transversal por amostra de conveniência para desenvolvimento de aplicativo móvel para uso no ensino e prática de profissionais médicos.

O aplicativo foi desenvolvido no Laboratório de Inovações Tecnológicas do Centro Universitário Christus, Campos Parque Ecológico, localizado a Rua João Adolfo Gurgel,133, em Fortaleza, Ceará. A Unichristus é uma universidade privada situada na cidade de Fortaleza, no Ceará. O curso de Medicina existe há 15 anos, e um de seus destaques é o fato de ter sido pioneiros no Ceará a utilizar as metodologias ativas de aprendizagem, apresentando um plano de ensino híbrido, que envolve metodologias como exposições dialogadas, sala invertida, laboratório de simulação, problem based learning (PBL), team based learning (TBL) e vivências clínicas.

Os questionários foram aplicados em salas de tutoria com professores e alunos também no Centro Universitário Christus. A população do estudo foi escolhida aleatoriamente entre os 120 alunos do oitavo semestre. Participaram do estudo 24 alunos do curso de medicina da Unichristus. Tais alunos já haviam cursado disciplinas de Nefrologia, Infectologia e Pediatria para a inclusão na pesquisa, além de afirmarem ter experiência no uso de aplicativos de celular (como Whatsapp ou Waze).

12 Professores tutores do quinto semestre também foram escolhidos aleatoriamente para avaliar o aplicativo.

Seriam excluídos todos aqueles que por algum motivo não se sentissem à vontade a participar de tal atividade, mas não houve qualquer recusa durante nossas solicitações.

Para a construção do aplicativo houve a participação de uma equipe multidisciplinar composta por: um professor de Medicina da área de Infectologia e outro da área de Nefrologia Pediátrica, um analista de sistemas, um programador e um designer de interfaces. Considerando os diferentes perfis de profissionais envolvidos no desenvolvimento e com o objetivo de se produzir uma aplicação mais próxima às necessidades dos usuários finais, optou-se pela utilização da metodologia Co-Design (Millard et al., 2009), divida em 5 etapas: escopo, compreensão compartilhada, Brainstorming (Desenvolvemos as interfaces da aplicação), refinamento e implementação. 
Foi estabelecido fluxo de atividades que envolveu reuniões regulares entre orientador, orientando deste estudo e equipe de desenvolvimento do software. Foi construída, em Power Point ${ }^{\circledR}$, espelhos para as telas do futuro aplicativo e a cada reunião, novas solicitações eram definidas, até a criação do protótipo do aplicativo. Sendo definida uma interface amigável e intuitiva, com conteúdo educacional aplicado à prática.

$\mathrm{O}$ aplicativo Pediálise contém questões importantes frente à abordagem da insuficiência renal crônica e diálise pediátrica, planejadas pelos pesquisadores com experiência na área. Após a finalização do aplicativo, sem erros aparentes, foi aplicada com dados de caso clínico de paciente fictício sujeitos a diálise pediátrica.

Para o desenvolvimento de caso clínico fictício e de um tutorial para uso do aplicativo Pediálise, promovemos reuniões com a equipe envolvida para estabelecer os objetivos de aprendizagem do teste com o aplicativo e escrevemos caso clínico de paciente fictício com patologia habitual em nefrologia pediátrica. No caso estavam presentes descrição do quadro clínico, anamnese, detalhes do exame físico, exames laboratoriais e de imagem pertinentes e, ao final, três questões clínicas a serem respondidas com o uso do aplicativo.

Para facilitar o manuseio do aplicativo, desenvolvemos tutorial básico em ferramenta Power Point ${ }^{\circledR}$ que demonstrava todas as funcionalidades presentes.

O estudo foi conduzido durante a aula de tutoria de alunos de Medicina do Centro Universitário Christus no ano de 2021. Participaram da pesquisa 37 indivíduos, sendo: 12 professores tutores do $5^{\circ}$ semestre; 9 alunos do $8^{\circ}$ semestre que utilizaram o aplicativo e 16 alunos do $8^{\circ}$ semestre que responderam ao caso sem o uso do aplicativo (controles),conforme o ilustrado na Figura 1.

O estudo de caso ocorreu em salas de aula de tutoria, no ambiente da própria turma em que o estudo foi aplicado, em dias letivos. Os grupos de teste do aplicativo utilizaram tablets com a última versão do App instalada pelo setor de informática (na plataforma Android) para a resolução do caso. O grupo controle foi realizado com alunos do oitavo semestre, que já tinham cursado as cadeiras de nefrologia, pediatria e infectologia e que resolveram o mesmo caso pelo método tradicional, sem uso o aplicativo.

Todas as repostas dos alunos foram armazenadas em bases de dados na nuvem computacional da Google®.

Para a avaliação da aplicação foi elaborado um questionário dividido em quatro partes. Uma questão inicial buscou identificar o nível de distração que o aluno poderá ter ao usar o celular em sala de aula. A segunda parte continha questões que objetivavam verificar a usabilidade e facilidade de uso do aplicativo. A terceira parte estava relacionada a perguntas voltadas para se obter a percepção dos alunos sobre a utilidade da aplicação para a aprendizagem deles. Por fim, a última parte continha uma questão aberta que com o objetivo de coletar os pontos negativos e positivos do aplicativo e as sugestões de melhorias.

Para se obter o grau de usabilidade do aplicativo, foi utilizado o questionário SUS (System Usability Scale), (Brooke, 1996) na versão traduzida para o português (Tenorio et al., 2011). O SUS visa medir a usabilidade de diversos produtos e serviços, especialmente utilizado para avaliação de sistemas tecnológicos como websites, hardware, aplicações móveis, etc. Ele se caracteriza como método de fácil aplicação onde cada questão contém cinco opções de respostas que seguem a escala Likert de 5 pontos (de Discordo Totalmente a Concordo Totalmente). Através do SUS é possível obter informações sobre a facilidade de uso (Usabilidade) e a simplicidade para se aprender a usar a aplicação (Capacidade de Aprendizado). Seguem abaixo as 10 primeiras questões do instrumento que foi utilizado:

1. Acho que gostaria de usar esse sistema com frequência.

3. Achei o sistema desnecessariamente complexo.

4. Achei o sistema fácil de usar.

5. Achei que seria necessário o apoio de um técnico para poder usar este sistema. 
6. Achei que as funções do sistema estavam bem integradas.

7. Achei este sistema muito inconsistente.

8. Imagino que a maioria das pessoas aprenderiam a usar esse sistema rapidamente.

9. Achei o sistema muito complicado de usar.

10. Senti-me muito confiante ao usar o sistema.

11. Precisei aprender várias coisas antes de continuar usando este sistema.

A terceira parte do instrumento de avaliação será composta por questões que terão como base o modelo de aceitação de tecnologia de Davis (Venkatesh \& Davis, 2000), em inglês Technology Acceptance Model - TAM. São elas:

1. Acho que o uso de dispositivos digitais durante a aula expositiva com aplicativos educacionais pode diminuir seu uso para fins não relacionados com o conteúdo administrado.

2. Acredito que o uso de dispositivos digitais com aplicativos educacionais pode aumentar o tempo de atenção do aluno em uma aula expositiva.

3. Acho que o uso interativo de dispositivos digitais com aplicativos educacionais em aula expositiva melhora a compreensão do conteúdo administrado.

4. Acho importante a utilização da tecnologia digital educacional em aulas expositivas.

Para avaliação da utilidade do aplicativo Pediálise, elaboramos questionário a ser respondido em escala Likert:

1. Os critérios analisados pelo App poderão ajudar no acompanhamento de insuficiência renal crônica e diálise em pediatria.

2. A recomendação final de antibioticoterapia de acordo com o histórico e quadro clínico atual do paciente poderá ajudar no uso racional de antibióticos

3. Acredito que a padronização através de um passo-a-passo proposto pelo App pode auxiliar no acompanhamento e tratamento adequado da insuficiência renal crônica e da diálise e suas complicações pelos estudantes e médicos.

4. Parece uma tecnologia útil para o diagnóstico, acompanhamento e tratamento adequado da insuficiência renal crônica com diálise pediátrica

5. O aplicativo ajudou-me a responder as questões de acordo com o caso clínico proposto 
Figura 1. Fluxograma para aplicação dos Casos entre as Turmas Controle e as que utilizarão o App para auxílio nas condutas frente à diálise pediátrica.

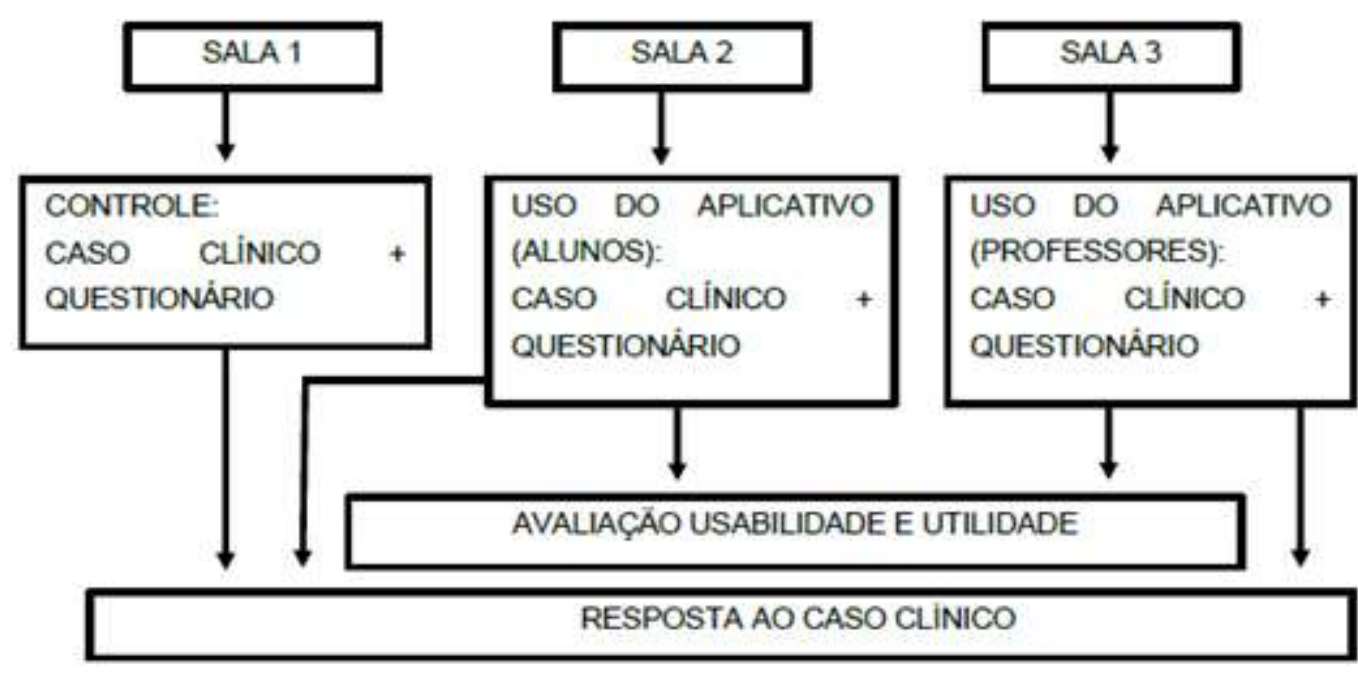

Fonte: Autores.

Para análise estatística, os dados foram tabulados no Microsoft Excel para Windows®, seguido de exportação para o software Statistical Package for the Social Sciences (SPSS) versão 20.0 (IBM) no qual foi adotada uma confiança de 95\%, com um valor $\mathrm{P}$ menor que 0,05 considerado estatisticamente significativo. Foram calculadas as médias e desvio-padrão, as frequências absolutas e percentual e os coeficientes de consistência interna (alfa de Cronbach) para todos os itens da escala SUS.

Os direitos e os aspectos éticos foram assegurados pela resolução 466\12 do Conselho Nacional de Saúde e a declaração de Helsinque.

O projeto foi submetido via plataforma Brasil na data 27/02/20 e aprovado pelo Comitê de Ética do Centro Universitário Christus, conforme o parecer número 3945667 CAAE 29440320.0.0000.5049 no dia 31/03/20. Os estudantes e professores da pesquisa participaram de forma voluntária, após assinatura do Termo de Consentimento Livre e Esclarecido

Nenhuma informação obtida ou gerada por meio da utilização do aplicativo, incluindo dados clínicos ou de identificação de pacientes, ou mesmo de usuários, foi coletada pelo software ou enviada via eletrônica para coleta, pesquisa ou venda para bancos de dados.

\section{Resultados}

Desenvolvemos o aplicativo denominado Pediálise para estudo de doença renal crônica e acessos para diálise pediátrica e suas complicações.

Na tela inicial do Pediálise (Figura 2) conseguimos ver os pacientes já cadastrados para escolher acessar seus dados ou clicar em ícone de "+" no canto direito da tela para adicionar novo paciente, sendo o usuário direcionado para uma aba de inserção de dados do paciente: Nome, data de nascimento, número de prontuário, nome da mãe, nome do pai; endereço, telefone, indicação de diálise, tipo sanguíneo e indicação de terapia substituição renal. 
Figura 2. Telas do Aplicativo Pediálise.

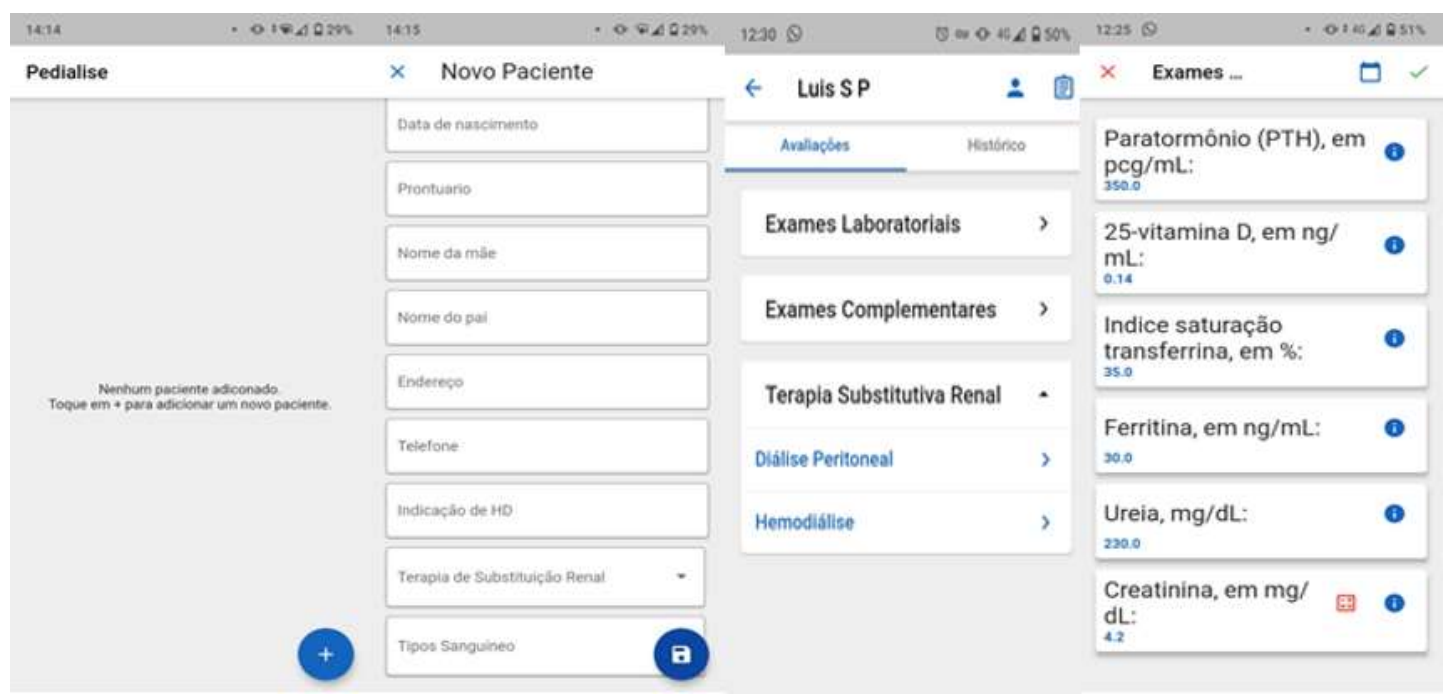

Fonte: Autores.

Ao salvar os dados, surge uma nova tela onde podemos escolher inserir nova avaliação ou ver histórico já cadastrado desse paciente. A nova avaliação permite escolha em inserção de dados de novos exames laboratoriais, outros exames complementares ou início de terapia substitutiva renal (subdivida em diálise peritoneal ou hemodiálise).

Quando escolhido ícone de exames laboratoriais, surge espaço para inserção de diversos exames importantes relacionados ao tratamento da doença renal crônica como: Paratormônio, 25-vit-D, Ferritina, Índice Saturação Transferina, Ureia, Creatinina e ao lado de cada item existe ícone de “i” onde é possível consultar valores de referência de cada teste.

Ao lado do ícone Creatinina vemos um ícone de calculadora. Quando escolhemos essa opção, uma calculadora permite o cálculo do Clearence de Creatinina estimado para sexo, idade e estatura do menor através da fórmula de Schwartz (Schwartz et al., 1984) a mais utilizada em pacientes pediátricos.

Ao clicar no ícone exames complementares, surge nova tela onde exames de imagem e outros importantes para pacientes com doença renal crônica são exibidos: Ultrassom de rins e vias urinárias, Uretrocistografia miccional, Estudo urodinâmico, ultrassom doppler de vasos, Sorologias, etc. Conseguimos assinalar se o exame é normal, alterado (e inserir qual a alteração) ou se não foi realizado ainda.

Ao clicar no ícone terapia de substituição renal podemos escolher entre hemodiálise ou diálise peritoneal. Quando escolhemos diálise peritoneal podemos escolher local de inserção do cateter no abdômen (hemi-abdomen direito ou esquerdo) e se foram detectadas intercorrências durante o implante (obstrução, infecção de óstio, de túnel ou peritonite, sangramento, extrusão do cuff, etc).Já quando escolhemos hemodiálise, podemos escolher qual o tipo de cateter (duplo ou triplo lúmen, permcath), tamanho do cateter, localização (femoral, jugular, subclávia ou outro vaso), lateralização (direita ou esquerda) e se houveram intercorrências relacionadas a esse acesso (obstrução, infecção óstio, túnel ou corrente sanguínea).Ao lado do ícone tamanho do cateter existe ícone "i" onde podemos consultar tabela para escolha do tamanho de cateter baseado no peso do paciente.

$\mathrm{Na}$ aba Histórico temos acesso a todas as informações já inseridas para o paciente em questão, possibilitando um consolidado de informações com busca rápida que pode ser muito útil na escolha de qual cateter e localização escolher para um dado paciente.

Desenvolvemos um manual/ tutorial para utilização do aplicativo Pediálise, com a descrição do passo-a-passo necessário para inserção de dados e uso de todas as funcionalidades do programa. O manual foi desenvolvido em ferramenta Power Point ® e foi disponibilizado para uso em smartphone quando participantes estavam utilizando o aplicativo. 
Obtivemos respostas de 37 participantes, sendo divididos em 12 professores tutores do $5^{\circ}$ semestre; 9 alunos do $8^{\circ}$ semestre que utilizaram o aplicativo e 16 alunos do $8^{\circ}$ semestre que responderam ao caso sem o uso do aplicativo (controles). Todos os alunos do grupo controle já haviam cursado as cadeiras de nefrologia, pediatria e infectologia. Todos os participantes utilizam ou já utilizaram algum aplicativo de celular para fins profissionais ou educativos.

No grupo controle, a idade média foi de 22,8 anos (variando de 21 a 25 anos), sendo 14 mulheres e 2 homens. No grupo teste com alunos, a idade média foi de 23,1 anos (variando de 21 a 29 anos). No grupo teste com professores, a idade média foi de 37,2 anos (variando de 32 a 51 anos), sendo 10 mulheres e 2 homens (Quadro 1).

Quadro 1. Descrição da amostra de participantes da pesquisa.

\begin{tabular}{|l|l|l|r|r|c|}
\hline \multicolumn{1}{|c|}{ Grupos } & Caracteristicas & & $\begin{array}{c}\text { N }{ }^{\circ} \text { de } \\
\text { individuos }\end{array}$ & Média & $\%$ \\
\hline Grupo controle & Idade & & & 22,8 & \\
\hline (16 indivíduos) & Sexo & Masculino & 2 & & $12,50 \%$ \\
\hline & & Feminino & 14 & & $87,50 \%$ \\
\hline Grupo Teste Alunos & Idade & & 2 & 23,1 & \\
\hline (9 individuos) & Sexo & Masculino & 7 & & $77,70 \%$ \\
\hline & & Feminino & & & \\
\hline $\begin{array}{l}\text { Grupo Teste } \\
\text { Professores }\end{array}$ & Idade & & & 37,2 & \\
\hline (12 inidivíduos) & Sexo & Masculino & 2 & & $16,60 \%$ \\
\hline & & Feminino & 10 & & $83,40 \%$ \\
\hline
\end{tabular}

Fonte: Autores.

Para avaliação da pontuação final do SUS, observamos que dois professores deixaram respostas em branco, sendo excluídos para a análise final.

A pontuação na escala SUS final foi de 77 no grupo dos alunos (variando entre 50 a 90), sendo considerado como excelente. No grupo dos professores a escala SUS final foi de 72,5 (variando entre 32,5 e 100), sendo considerado como bom. Avaliando os dois grupos, a escala SUS final foi de 74,5, sendo classificada como excelente.

A Tabela 1 resume estatisticamente a análise sobre Usabilidade da aplicação Pediálise utilizando as questões baseadas na escala SUS (gráfico 1 e tabela 3) para verificação da facilidade de uso do sistema. Os resultados demonstram que a aplicação recebeu uma boa avaliação de usabilidade, obtendo escore SUS médio igual a 74,5 (com desvio padrão de 19,9). Estudos apontam o valor 70,0 como sendo o escore médio SUS mínimo para se considerar um sistema com um bom nível de usabilidade (Bangor et al., 2009; Sauro \& Lewis, 2012). Além disso, também se pode afirmar, com 95\% de confiança, que o escore SUS para essa população está entre 65,2 e 83,8 (com margem de erro igual a 9,3).

Tabela 1. Resumo da análise sobre Usabilidade da aplicação Pediálise com N =20.

\begin{tabular}{c|c}
\hline Variável & Valor \\
\hline Tamanho da Amostra & 20 \\
\hline Escore Médio SUS & 74,5 \\
\hline Intervalo de Confiança & $65,2-83,8$ \\
\hline Margem de Erro & 9,3 \\
\hline Nível de Confiança & $95 \%$ \\
\hline Desvio Padrão & 19,9 \\
\hline Confiabilidade & 0,86
\end{tabular}


Gráfico 1. Análise individual de Usabilidade da aplicação Pediálise na escala SUS.

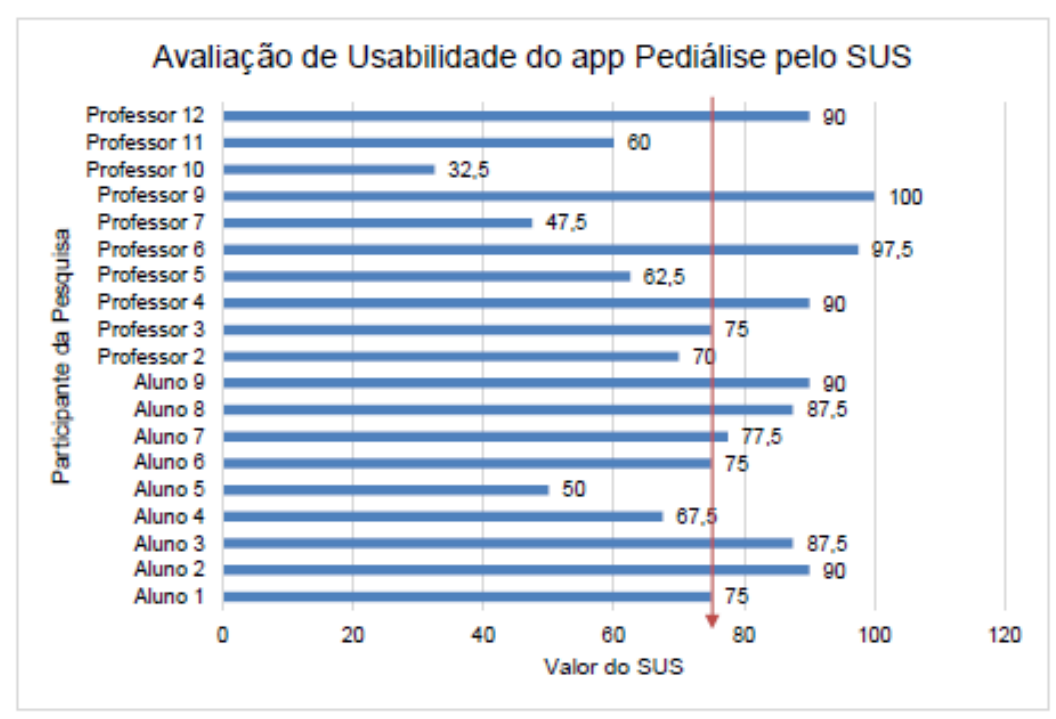

Fonte: Autores.

Para atestar a confiabilidade dos dados obtidos, utilizou-se o coeficiente alfa de Cronbach (Bonett e Wright, 2015). O maior valor possível para esse coeficiente é 1,00, sendo 0,70 considerado o limite inferior para uma confiabilidade interna aceitável (Sauro, 2011). O coeficiente alfa de Cronbach obtido nesse estudo foi de 0,86, caracterizando a amostra com um bom nível de confiabilidade.

Tabela 2. Resumo das respostas do SUS com percentual de cada resposta de usuários que testaram o app Pediálise.

\begin{tabular}{|c|c|c|c|}
\hline Escala Sus & & & \\
\hline Pergunta & Resposta & & $\%$ \\
\hline 1. Eu gostaria de usar esse sistema frequentemente & & 2 & $4,76 \%$ \\
\hline & & 3 & $14,28 \%$ \\
\hline & & 4 & $28,50 \%$ \\
\hline & & 5 & $52,30 \%$ \\
\hline 2. Eu achei o aplicativo desnecessariamente complexo & & 1 & $33,30 \%$ \\
\hline & & 2 & $28,50 \%$ \\
\hline & & 3 & $4,76 \%$ \\
\hline & & 4 & $23,80 \%$ \\
\hline & & 5 & $9,50 \%$ \\
\hline 3. Eu achei o aplicativo fácil para usar & & 2 & $9,50 \%$ \\
\hline & & 3 & $9,50 \%$ \\
\hline & & 4 & $23,80 \%$ \\
\hline & & 5 & $57,10 \%$ \\
\hline 4. Eu acho que precisaria de suporte tecnico frequente para utilizar esse aplicativo & & 1 & $14,28 \%$ \\
\hline & & 2 & $33,30 \%$ \\
\hline & & 3 & $14,28 \%$ \\
\hline & & 4 & $4,76 \%$ \\
\hline & & 5 & $23,80 \%$ \\
\hline & não respondeu & & $9,50 \%$ \\
\hline 5. Eu achei que as diversas funções do aplicativo foram bem integradas & & 2 & $9,50 \%$ \\
\hline
\end{tabular}




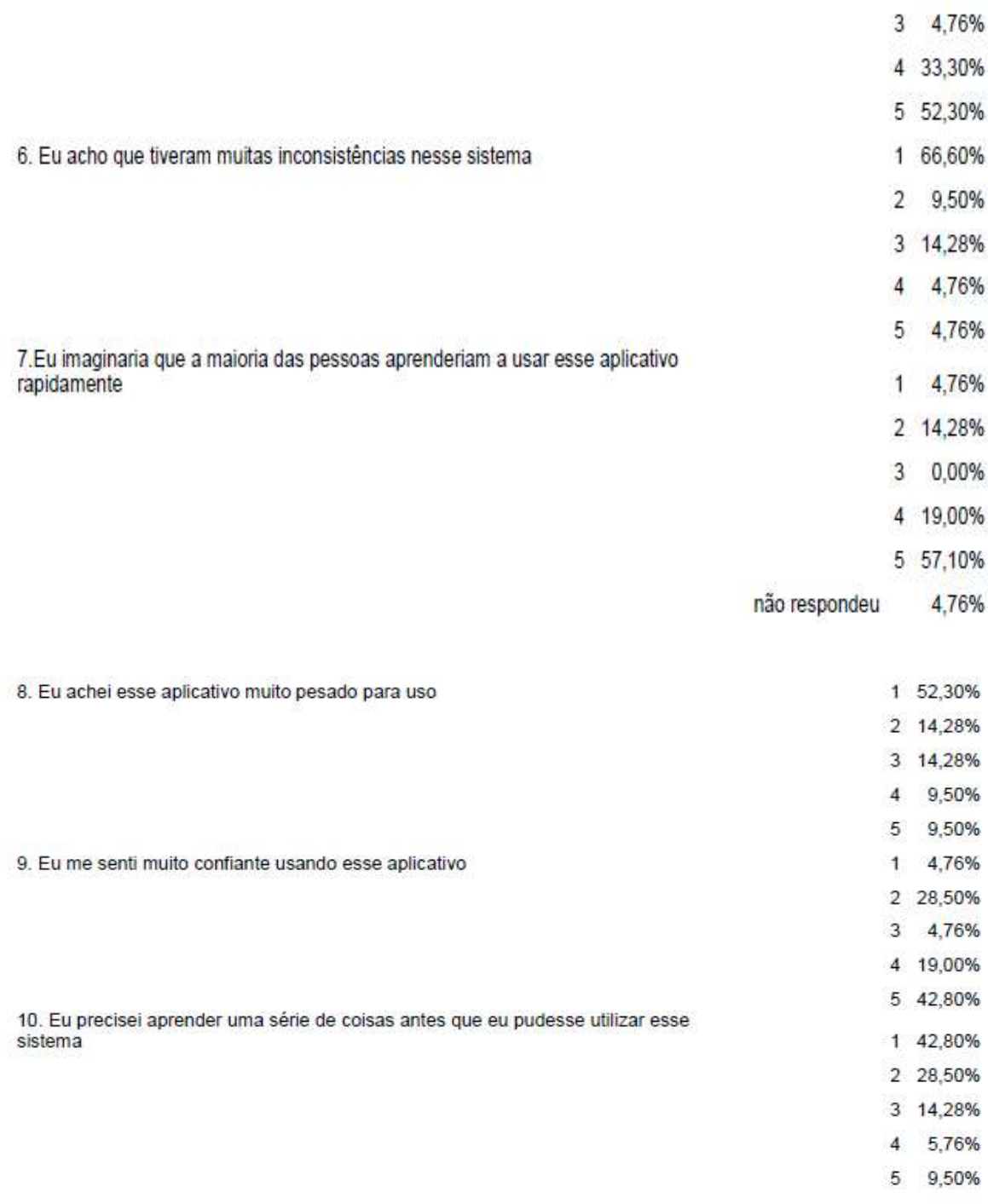

Fonte: Autores.

Com o uso do aplicativo, alunos e professores tiveram elevado percentual de acerto das questões solicitadas. Na questão 1 (Calcule o Clearence de Creatinina estimada para o paciente dada a idade do menor, ideal uso da fórmula de Schwartz), o grupo teste respondeu corretamente em $100 \%$ das vezes no grupo dos alunos e em 91,6 \% no grupo dos professores. Já no grupo controle, $33,3 \%$ dos alunos conseguiram fornecer resposta correta.

Na questão 2 (Qual tamanho de cateter seria corretamente indicado?) o grupo teste respondeu corretamente em 100\% no grupo alunos e também no grupo professores. Já no grupo controle, nenhum aluno conseguiu fornecer a resposta correta.

Na questão 3 (Qual antibiótico deverá ser utilizado, duração do tratamento e dose ajustada?), o grupo teste respondeu corretamente qual antibiótico a ser utilizado em $100 \%$ no grupo alunos e $83,3 \%$ no grupo dos professores. Já no grupo controle, houve acerto na escolha do antibiótico em 33,3\%, na dose em 13,3\% e não houveram acertos na duração do tratamento.

A maioria das respostas dos participantes do grupo controle foi "Não sei". No questionamento sobre qual o antibiótico deveria ser utilizado, os alunos elencaram outros antimicrobianos, mas falharam em indicar o antibiótico ideal em $66 \%$ das respostas. Algumas questões como cálculo do clearence e tamanho do cateter e ser escolhido precisam de conhecimento de valores de referência específicos para faixa etária e peso, sendo difícil que alunos e, possivelmente, também profissionais de saúde, respondam adequadamente a essas questões sem consulta. 
Percebemos também que nenhum dos alunos do grupo teste responderam ao item dose do antibiótico e que 5 dos 9 alunos também deixaram em branco a questão sobre duração do tratamento. Não ficou claro se houve esquecimento ou se não sabiam a resposta.

Sobre a utilidade do aplicativo Pediálise podemos observar nas tabelas que houve pouca diferença entre os grupos alunos e professores.

No grupo dos professores, $75 \%$ concordaram totalmente com todas as perguntas no questionário de utilidade (gráfico 3). No grupo teste dos alunos, houve concordância total variando de 66,6\% a 77,7\%, com concordância média de 75,9\%.

Gráfico 3. Análise das respostas sobre Utilidade do aplicativo Pediálise de alunos da Unichristus.

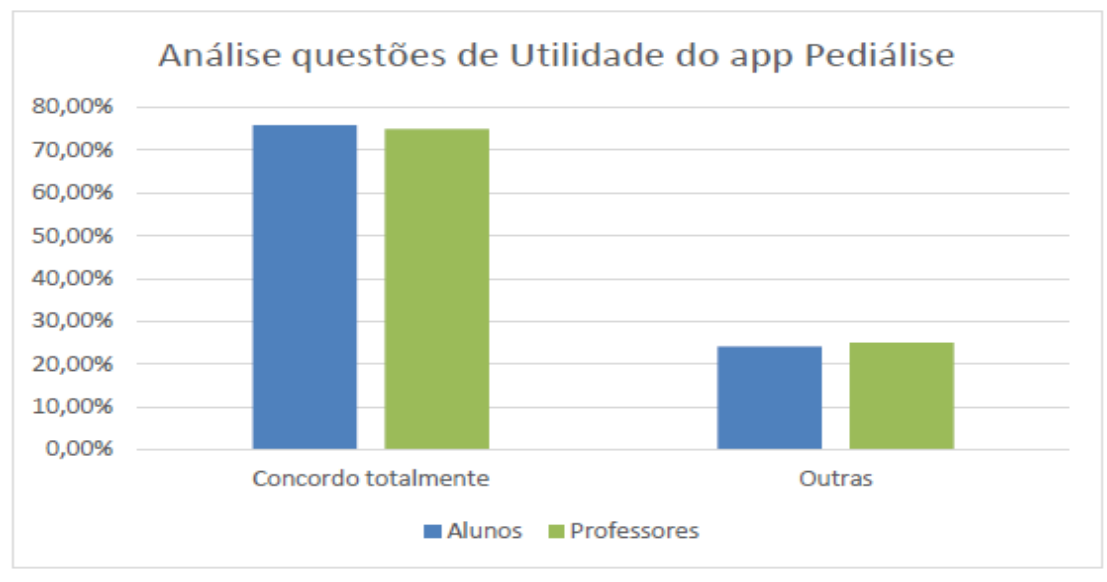

Fonte: Autores.

Além das questões objetivas com a avaliação pela escala SUS, solicitamos aos participantes que fornecessem sugestões para melhoria do aplicativo e indicar os pontos positivos e negativos. As respostas confirmam a boa aceitação da aplicação móvel, ressaltando-se utilidade e facilidade de manuseio. A maioria das sugestões sugere aba com tutorial no aplicativo.

A seguir, são listadas todas as opiniões coletadas sobre o aplicativo.

\section{Pontos positivos:}

\begin{tabular}{|c|c|c|c|}
\hline - & Aluno 1: "fácil manuseio" & $\cdot$ & Professor 4: "informações objetivas e completas" \\
\hline - & Aluno 2: "muito útil" & - & Professor 5: "ajudarão os pacientes bastante" \\
\hline$\cdot$ & Aluno 3: "fácil manuseio" & $\cdot$ & Professor 6: "praticidade, segurança, detalhado" \\
\hline - & Aluno 4: "será útil para profissionais" & - & Professor $7:$ "acessivel" \\
\hline - & Aluno $7:$ "Bastante útil" & - & Professor 8: "intuitivo e didático" \\
\hline - & Aluno 8: "Facilidade de uso" & - & Professor 9: "praticidade, detalhamento" \\
\hline - & Professor 1: "útil uso da tecnologia" & $\cdot$ & Professor 10: "informações fornecidas" \\
\hline - & Professor 2: "rapidez, facilidade para inserção de dados" & $\cdot$ & Professor 12: "auxilia em cálculos e dúvidas terapêuticas \\
\hline - & Professor 3: "rapidez e fácil de usar" & & \\
\hline
\end{tabular}




\section{Pontos negativos:}

\begin{tabular}{l|l}
\hline Aluno 1: "não encontrei alguns tópicos (ATB)" & Professor 5: "achei que precisa ter aba com tutorial" \\
\hline Aluno 2: "não encontrei dose do medicamento" & Professor 7: "não soube usar as informações de inicio" \\
\hline Aluno 4: "Dificuldade inicial para manusear" & Professor 8: "nenhum" \\
\hline $\begin{array}{l}\text { Professor 1: "achei confuso, tive que fazer a mesma coisa } \\
\text { várias vezes" }\end{array}$ & Professor 10: "complexidade" \\
\hline $\begin{array}{l}\text { Professor 2: "pode haver termos que não são comuns ao } \\
\text { generalista" }\end{array}$ & Professor 12: "necessita de explicação, mas é básica" \\
\hline
\end{tabular}

\section{Sugestões:}

\begin{tabular}{l}
$\begin{array}{l}\text { Professor 1: "Precisa ajustar alguns bugs, não gravou a } \\
\text { resposta. Inserir pergunta mais direta: quem calculou - } \\
\text { clearence?" }\end{array}$ \\
\hline $\begin{array}{l}\text { Professor 5: "Pode ter aba com tutorial, não achei muito } \\
\text { intuitivo" }\end{array}$ \\
\hline Professor 7: "Explicar uso com tutorial no inicio do app"
\end{tabular}

\section{Discussão}

Desde o aparecimento da telefonia na década de 1980, a utilização de celulares cresceu enormemente. Atualmente estima-se que existam cerca de 7 bilhões de dispositivos, $80 \%$ deles sendo smartphones. Os profissionais de saúde utilizam bastante de toda essa tecnologia e 50\% usam aplicativos médicos para auxiliar sua prática clínica. Dessa forma, esses aplicativos podem auxiliar no processo de ensino/aprendizagem e na tomada de decisão frente ao paciente real. (Michard, 2019)

Nos grupos que utilizaram o aplicativo Pediálise observamos um aumento importante no índice de acertos das questões do caso clínico. Em alguns itens houve 100\% de respostas corretas no grupo teste e nenhum acerto no grupo controle.Essa análise nos leva a concluir que a utilização do App Pediálise foi importante para guiar as respostas de conduta no paciente renal crônico pediátrico. Embora, devamos relembrar que algumas questões como cálculo do clearence e tamanho do cateter a ser escolhido precisam de conhecimento de valores de referência específicos para faixa etária e peso, sendo difícil que alunos e, possivelmente, também profissionais de saúde, respondessem adequadamente a essas questões sem consulta.

O App Pediálise aponta para um grande benefício na conduta terapêutica do paciente pediátrico portador de IRC. Como o App foi alimentado com as tabelas de antibióticos padronizados e com fluxograma de uso, ele poderá facilitar as escolhas corretas e evitar os erros de conduta que impactam diretamente na segurança do paciente.

Em estudo semelhante de desenvolvimento e validação de um aplicação para auxiliar no processo de ensino e aprendizagem de construção de um retalho cutâneo por estudantes de medicina, o grupo de aprendizagem assistida por app teve um desempenho superior tanto em avaliação geral global $(\mathrm{p}=0,017)$ e resultados pós-teste (p <0,001). ( Sena, 2013).

No Canadá, estudantes do terceiro ano de medicina que usaram aplicativo móvel para manobra de reposicionamento de partículas de Epley, um tratamento eficaz para a vertigem posicional paroxística benigna, obtiveram pontuação significativamente maior no desempenho em comparação com o grupo controle $(\mathrm{P}<0,0001)$. Esses resultados mostram o potencial da aprendizagem móvel como ferramenta auxiliar no ensino médico. (Organ, 2015).

O aplicativo desenvolvido foi bem avaliado em relação a usabilidade, obtendo nota excelente no grupo teste de alunos, boa no grupo teste dos professores e escala SUS final de 74,5 com desvio padrão de 19,5- considerada excelente quando 
avaliamos os dois grupos em conjunto. Estudos apontam o valor 70,0 como sendo o escore médio SUS mínimo para se considerar um sistema com um bom nível de usabilidade (Bangor et al., 2009; Sauro \& Lewis, 2012).

Em uma revisão de escopo recente com 133 artigos sobre métodos de avaliação de aplicativos em saúde, o questionário SUS foi o método mais utilizado apesar de não identificar os problemas que precisam ser resolvidos, sendo sugerido uso de métodos qualitativos (Maramba,2019).

A avaliação das respostas sobre pontos positivos é bem interessante porque complementa a avaliação SUS. Ambos os grupos ressaltam a facilidade, praticidade de utilização do App e o quanto o mesmo poderá ser útil como ferramenta de diagnóstico e conduta.

As avaliações negativas citaram não encontrar alguma informação no aplicativo (mesmo que essa informação estivesse sim disponível) e sugeriram a incorporação de tutorial ao aplicativo. Nosso tutorial foi disponibilizado em outra ferramenta e precisava ser consultado com o smartphone do usuário, talvez, o fato de necessitar de duas plataformas tornou o processo desconfortável e alguns usuários acabaram não consultando o tutorial mesmo sendo informados de que ele estava disponível. As sugestões também abordam o tema presença de tutorial e solicitam que o esse manual esteja dentro do aplicativo para facilitar sua consulta.

Broekhius (2019), em estudo comparando instrumentos de avaliação de usabilidade para tecnologias na saúde, concluiu que listar os problemas de usabilidade pensando em voz alta é uma das ferramentas mais úteis e que utilizar o SUS como método de avaliação isoladamente não é recomendado (Broekhius, 2019).

Reeder (2019) obteve excelente pontuação média SUS de 92,5 ao avaliar um aplicativo para diagnóstico de infecção urinária, entretanto, foi através de entrevistas, pensar em voz alta, feedbacks que houveram recomendações de mudanças de desing de interface. (Reeder ,2019)

O aplicativo Pediálise tem o potencial de ajudar alunos, internos e residentes no estudo de temas relacionados à nefrologia, nefrologia pediátrica e infectologia ao oferecer uma fonte rápida e atualizada de dados sobre doença renal crônica em pediatria. Além do baixo custo para utilização do aplicativo Pediálise, temos algumas vantagens como: rápido acesso à informação (consulta rápida de protocolos para uso de antibioticoterapia, doses e duração do tratamento com determinado antibiótico, tamanho de cateter indicado, etc); portabilidade (inerente ao uso de aplicativos em smartphones ou tablets, permitindo uso em diversidade de ambientes como sala de aula, beira leito, estudo guiado na residência, etc); facilidade para utilização (criamos aplicativo que permite fácil manuseio por indivíduos familiarizados com a tecnologia disponível com necessidade de tutorial básico); possibilidade de uso futuro do aplicativo como plataforma de inserção de dados e consulta para pacientes reais, facilitando trabalho de médicos em serviços de nefrologia pediátrica.

\section{Conclusão}

O desenvolvimento de Aplicativo Móvel para acompanhamento de acessos de diálise em pacientes pediátricos foi concluído. Cada etapa do processo permitiu que o aluno aprendesse sobre como conduzir intercorrências em pacientes renais crônicos em diálise.

Conseguimos validar a aplicação do Pediálise em grupos de alunos e professores do curso de medicina, demonstrando sua boa usabilidade e utilidade para fins educativos. Além de determinar sua usabilidade e facilidade, conseguimos boa avaliação qualitativa.

O novo produto tecnológico poderá ser utilizado como ferramenta complementar no ensino de nefrologia, nefropediatria e infectologia, visto que apresentou usabilidade satisfatória, e pode auxiliar nos diferentes cenários de metodologias ativas. Dessa forma, sugerimos continuar tal pesquisa aprimorando-a com uma amostra maior, visando a maior confiabilidade dos dados, além de melhorias e adaptações quanto as recomendações dadas nesse artigo. 


\section{Referências}

Arhuidese, I. J., Wanogho, J., Faateh, M., Aji, E. A., Rideout, D. A., \& Malas, M. B. (2020). Hemodialysis and peritoneal dialysis access related outcomes in the pediatric and adolescent population. Journal of pediatric surgery, 55(7), 1392-1399. https://doi.org/10.1016/j.jpedsurg.2019.09.017

Bangor, A., Kortum, P., \& Miller, J. (2009). Professor-in-the-Practice. Journal of Usability Studies, 4, 114-123. https://uxpajournal.org/wpcontent/uploads/sites/7/pdf/JUS_Bangor_May2009.pdf

Bream P. R., Jr (2016). Update on Insertion and Complications of Central Venous Catheters for Hemodialysis. Seminars in interventional radiology, 33(1), 3138. https://doi.org/10.1055/s-0036-157254

Broekhuis, M., van Velsen, L., \& Hermens, H. (2019). Assessing usability of eHealth technology: A comparison of usability benchmarking instruments. International journal of medical informatics, 128, 24-31. https://doi.org/10.1016/j.ijmedinf.2019.05.001

Crabtree, J. H., \& Chow, K. M. (2017, January). Peritoneal dialysis catheter insertion. In Seminars in nephrology (Vol. 37, No. 1, pp. 17-29). WB Saunders.

de Sena, D. P., Fabricio, D. D., Lopes, M. H., \& da Silva, V. D. (2013). Computer-assisted teaching of skin flap surgery: validation of a mobile platform software for medical students. PloS one, 8(7), e65833. https://doi.org/10.1371/journal.pone.0065833

Echer, I. C. (2005). Elaboração de manuais de orientação para o cuidado em saúde. Revista Latino-Americana de Enfermagem, 13(5), 754-757. https://doi.org/10.1590/s0104-11692005000500022

Imani, P. D., Carpenter, J. L., Bell, C. S., Brandt, M. L., Braun, M. C., \& Swartz, S. J. (2018). Peritoneal dialysis catheter outcomes in infants initiating peritoneal dialysis for end-stage renal disease. BMC nephrology, 19(1), 231. https://doi.org/10.1186/s12882-018-1015-1

LaPlant, M. B., Saltzman, D. A., Segura, B. J., Acton, R. D., Feltis, B. A., \& Hess, D. J. (2018). Peritoneal dialysis catheter placement, outcomes and complications. Pediatric surgery international, 34(11), 1239-1244. https://doi.org/10.1007/s00383-018-4342-

Maramba, I., Chatterjee, A., \& Newman, C. (2019). Methods of usability testing in the development of eHealth applications: A scoping review. International journal of medical informatics, 126, 95-104. https://doi.org/10.1016/j.ijmedinf.2019.03.018

Michard, F., Barrachina, B., \& Schoettker, P. (2019). Is your smartphone the future of physiologic monitoring?. Intensive care medicine, 45(6), 869-871. https://doi.org/10.1007/s00134-018-5419-y

Millard, D., Howard, Y., Gilbert, L., Wills, G., Millard, D., Howard, Y., Gilbert, L., \& Wills, G. (2009, July 1). Co-design and Co-deployment Methodologies for Innovative m-Learning Systems. Eprints.soton.ac.uk; IGI Global. https://eprints.soton.ac.uk/267555/

Koch Nogueira, P. C., Santis Feltran, L. de, Camargo, M. F., Leão, E. R., Benninghoven, J. R., Gonçalves, N. Z., Pereira, LuizA., \& Sesso, R. C. (2011). Prevalência estimada da doença renal crônica terminal em crianças no Estado de São Paulo. Revista Da Associação Médica Brasileira, 57(4), 443-449. https://doi.org/10.1590/s0104-4230201100040002

Organ, B., Liu, H., \& Bromwich, M. (2015). An iPhone-assisted particle repositioning maneuver for benign paroxysmal positional vertigo (BPPV): a prospective randomized study. Journal of the American Board of Family Medicine : JABFM, 28(1), 118-120. https://doi.org/10.3122/jabfm.2015.01.120295 (PDF) SUS: A quick and dirty usability scale. (n.d.). ResearchGate. https://www.researchgate.net/publication/228593520_SUS_A_quick_and_dirty_usability_scale

Reeder, B., Drake, C., Ozkaynak, M., \& Wald, H. L. (2019). Usability Testing of a Mobile Clinical Decision Support App for Urinary Tract Infection Diagnosis in Nursing Homes. Journal of gerontological nursing, 45(7), 11-17. https://doi.org/10.3928/00989134-20190408-01

Rivacoba, M. Carolina, Ceballos, M. Luisa, \& Coria, Paulina. (2018). Infecciones asociadas a diálisis peritoneal en el paciente pediátrico: diagnóstico y tratamiento. Revista chilena de infectología, 35(2), 123-132. https://dx.doi.org/10.4067/s0716-10182018000200123

Sauro, J. (2011). A Practical Guide to the System Usability Scale: Background, Benchmarks \& Best Practices. In Google Books. Measuring Usability LLC. https://books.google.com.br/books/about/A_Practical_Guide_to_the_System_Usabilit.html?hl=es\&id=BLOkKQEACAAJ\&redir_esc=y

Sauro, J., \& Lewis, J. R. (2016). Quantifying the User Experience: Practical Statistics for User Research. In Google Books. Morgan Kaufmann. https://books.google.com.br/books?hl=pt-BR\&lr=\&id=USPfCQAAQBAJ\&oi=fnd\&pg=PP1\&dq=Sauro

Schwartz, G. J., Feld, L. G., \& Langford, D. J. (1984). A simple estimate of glomerular filtration rate in full-term infants during the first year of life. The Journal of pediatrics, 104(6), 849-854. https://doi.org/10.1016/s0022-3476(84)80479-5

Schwartz, G. J., \& Gauthier, B. (1985). A simple estimate of glomerular filtration rate in adolescent boys. The Journal of pediatrics, 106(3), 522-526. https://doi.org/10.1016/s0022-3476(85)80697-1

Szeto, C.-C., Chow, K.-M., Wong, T. Y.-H., Leung, C.-B., Wang, A. Y.-M., Lui, S.-F., \& Li, P. K.-T. (2002). Feasibility of Resuming Peritoneal Dialysis after Severe Peritonitis and Tenckhoff Catheter Removal. Journal of the American Society of Nephrology, 13(4), 1040-1045. https://doi.org/10.1681/asn.v1341040

Tenório, J. M., Cohrs, F. M., Sdepanian, V. L., Pisa, I. T., \& Marin, H. de F. (2010). Desenvolvimento e Avaliação de um Protocolo Eletrônico para Atendimento e Monitoramento do Paciente com Doença Celíaca. Revista de Informática Teórica E Aplicada, 17(2), 210-220. https://doi.org/10.22456/2175-2745.12119

Venkatesh, V., \& Davis, F. D. (2000). A Theoretical Extension of the Technology Acceptance Model: Four Longitudinal Field Studies. Management Science, 46(2), 186-204. https://doi.org/10.1287/mnsc.46.2.186.11926

Weber, S., Martins, R., \& Martins, N. (2017). Risk factors for respiratory complications after adenotonsillectomy in OSA children. Sleep Medicine, 40, e344e345. https://doi.org/10.1016/j.sleep.2017.11.1016 